\title{
Use of Self-Reporting Questionnaires to Evaluate Augmented Paper Maps for Group Navigation
}

\author{
Andreas Komninos ${ }^{1}$, Jeries Besharat ${ }^{2}$ and John Garofalakis ${ }^{2}$ \\ ${ }^{1}$ University of Strathclyde, Glasgow, United Kingdom \\ andreas.komninosestrath.ac.uk \\ ${ }^{2}$ University of Patras / RACTI, Rio, Patras, Greece \\ $\{$ besarat, garofala\}@ceid.upatras.gr
}

\begin{abstract}
One popular and widely use of augmented reality based application, is the projection of points of interests on top of the phones' camera view. In this paper we discuss the implementation of an AR application that acts as a magic lens over printed maps, overlaying POIs and routes. This method expands the information space available to members of groups during navigation, partially mitigating the issue of several group members trying to share a small screen device. Our work complements existing literature by focusing on the navigation tasks and by using self-reporting questionnaires to measure affective state and user experience. We evaluate this system with groups of real tourists in a preliminary field trial and report our findings.
\end{abstract}

Keywords: Augmented reality $\cdot$ group navigation $\cdot$ mobile maps $\cdot$ tourism $\cdot$ augmented maps.

\section{$1 \quad$ Introduction}

Many location based mobile applications today allow users to discover the location of Points of Interest (POIs) in a city. Teevan et al. [20] found that the most common reason of searching for a POI on a mobile device was to get directions to that POI. Typically, map exploration and navigation with mobile devices are done using the small screen space available. While navigation applications for single users are well developed and manage to empower users sufficiently, in many situations users do not navigate alone, but as part of a group. In such cases, the convergence of multiple users over a single small-screen device is problematic, as the information display area is too small to be viewed by all members of the group. Hence, collaborative navigation, where multiple users can offer their interpretation of instructions or make decisions on routes to take, is difficult when using mobile devices. In this paper we describe an alternative approach for group navigation, based on the augmentation of a physical paper map. We believe that such a hybrid system can be shared more easily within a group. Our prototype, which is called HoloPlane, shows POIs collected from the Foursquare API. HoloPlane uses real-time and historical data from social networking 
services (as in, e.g. [7]), to display these POIs in a manner that allows users to understand their popularity under the current temporal context. Users can see their own location on the map as a virtual marker and can select POIs to navigate to. Routes are displayed as a set of virtual lines, aligned with the street structure on the printed map. Our paper presents results from a preliminary field evaluation study on the prototype's use during group navigation tasks, using a validated questionnaire approach to evaluation that has not been employed in related research in the past.

\section{$2 \quad$ Related Work}

Traditionally, paper maps have played major roles in conveying spatial information and guiding people around in space. However, this standard experience could be enhanced and improved, as it is shown that augmented paper maps could be used to develop interactive paper maps that will provide added values services for tourists [13]. Stroila et al. [17] demonstrated an AR navigation application, which allows users to interact with transit maps in public transit locations and vehicles.

In [12] the acceptance and usability of an AR system that provides pedestrian navigation through a combination of mobile devices and public displays are studied, but with focus on single users and not collaborative use, as does most literature on this subject. The effectiveness of navigating to POIs with an AR browser and a 2D digital map interface is studied in [5]. It is found that although the use of AR with a digital map did not offer any advantages to performance, users preferred this mode strongly as it doesn't lock users into one type of interaction.

Other research has identified a range of issues concerning the use of AR and magic lens interaction. One is the dual-view problem in magic lens viewing [4], where users have to shift their attention between the mobile screen (magic lens) and the background augmented object. This causes difficulty in matching the mobile view with the background, as the mobile view appears at a different zoom level than the background object, hence posing cognitive difficulties to the user. A further issue arising from natural use of the mobile device, is the angular difference in the user's view of the background object and the device (e.g. the background object might be perpendicular to the ground as in the case of a fixed poster, while the mobile screen might be tilted in varying degrees, for example when the user holds the device up high to bring a tall part of the background object into view). A further issue concerns the size of the augmented object, in this case the background map.

In [6] it has been found that static peephole interfaces for maps are better than magic lenses, when the area of the map to be explored is small. As the size of the map increases, the differences even out and in fact, the magic lens interface becomes better to use in larger maps. The researchers obtained their findings using physical map sizes that are considerably larger than the typical handheld map (the smallest map used was $1.38 \mathrm{~m} \times 0.76 \mathrm{~m}$ ), making these findings applicable to large maps, of the kind that would be placed on a wall as a poster, or on a public display.

Finally, in [17], researchers find that item density can have an effect on how much time users spend looking at the background object, compared to the magic lens view. 
It was found that for low item density situations, users tended to focus more on the background object, confirming a previous experiment [16] where users focused more on the magic lens view, above a certain item density threshold.

In [3] the problems faced by tourists during holidays are outlined. The most common problems in an unfamiliar place, are what to do and when. The researchers explore how tourists solve their problems by relying on sharing the visit with other tourists ( $79 \%$ of leisure visits involve groups of two or more) and how they worked as a group by using digital technologies. The leisure activity seemed to be less important than the fact the tourists spent significant time with others. As a result, technologies that are woven into this sociality are likely to be used in preference to those that are not.

Reilly et al. [15] examined how groups of two share a single device during a collaborative indoor way finding activity. They developed two basic interfaces (one that combines map and textual descriptions and a textual interface that numbers the route description) in order to conduct the experiment. Their analysis on the results showed that the application's interface impacts the strategy users followed to complete the tasks. They found that some pairs heavily favored specific navigation strategies or sharing styles. This emphasizes the importance of group dynamic on the use of spatial applications.

A set of requirements for mobile indoor navigation systems that support collaborative path finding tasks is presented in [2]. The researchers observed and analyzed the actions participants performed such as walking, pointing, looking etc. and found that the pointing action, as a communication purpose, occurs much more in groups. Furthermore, the number of people involved in a group does complicate the process of completing the task. $76.4 \%$ of the participants stated that positioning and navigation signs helped them to find their target locations. There is very little relevant literature that discusses group navigation aspects using AR.

In [11], researchers augmented a map with POIs (but not navigation instructions) using a device as a magic lens as part of a pervasive game. They found that augmented maps offer advantages to groups as a collaboration tool, since groups that used them found it easier to establish common ground than groups of users who used only a digital map. Further work in [10] included use of multiple devices on the same map, which found that up to 2 devices are usable without causing issues. It was also shown that the ability to cluster and collaborate over the physical map enhanced the "feelgood" factor between group members. Neither [10] nor [11] seem to consider the dual-view problem, item density or map size for their effect on the usability of the AR maps. A significant shortfall of studies like [10] and [11] lies in the fact that only qualitative data was obtained by the researchers, in the form of interviews, coupled with their own direct observation. As pointed out in [1], these methods suffer from potential subjectivity bias and also from the researchers' own bias. This observation is highlighted again in [14], where it is found that the user's own context (i.e. whether they see themselves as a future user of the system under evaluation) can place a strong influence on the reported assessment of a system's usability. Hence, the findings in [10] and [11] provide a good insight into the usability of AR maps for collaborative use, but have to be considered as incomplete. As can be seen, the use of AR maps is an on-going subject of research with many unanswered questions, in both the cases of 
single users and collaborative use. Our main focus is, for this paper, to add to the small body of literature on user experience during AR-assisted group navigation, taking a different approach to evaluation (i.e. using selfreporting questionnaires that have been validated for effectiveness, to assess affective state and user experience). This element is entirely missing from existing literature, where results are largely based on direct observation and interviews.

\section{HoloPlane Interface}

Our prototype (HoloPlane) is built using the Qualcomm Vuforia SDK and Unity for Android. For the experiment described in this paper, our users were not asked to interact with the prototype features, except from viewing the route between the POIs that were pre-selected for them by the researchers. However, in the next section we provide an overview of how the prototype works, in order to demonstrate the full potential of our idea. The interface has been designed using several iterations of expertbased usability methods but the full UI design has not been tested with users for usability - this remains the subject of further work. Here we are primarily concerned with the concept of showing routes on an augmented paper map interface.

The printed map does not require special markings to be recognized by the device, as it is a recognition target in itself. When the application detects the map, it connects to our server and fetches the required POI information. This is overlaid on the map image along with a marker that shows the user's location. Once a user selects a POI to navigate to, the application downloads guidance instructions from the Google Directions API and renders route segments as virtual lines on the map. The application does not need to remain connected for its operation from this point onwards. The main interface of the application consists of five buttons that are placed on the top area of the screen and one informative panel on the bottom area of the screen. With this layout, we developed a service that conveys a range of contextual information to the user in a multi-layered view. The graphical elements in brackets are shown in Figure 1 (top). Layer 1: This layer is responsible for overlaying the POI information retrieved from our server. The POIs are presented as 3D that show, on all their surfaces, an icon that indicates the category the POI belongs to (1). The POIs are colour-coded to indicate whether they are popular or not, depending on the current time and day. The navigation route (if selected) is also shown (2), using virtual lines, aligned with the street structure on the map. The user's position, which is determined using the devices' GPS sensor, is also displayed as an arrow (3). Layer 2: This layer has all the UI control and is split in two sections, the top UI control bar (Figure 1 middle) and the bottom navigation panel (Figure 1 bottom). In the UI control bar, button (4) shows a list view with the names of all the POIs currently on the device screen. Users can select a POI from that list to identify it on the map. The application then "scales up" the POI cube briefly, to help the user identify it. This helps users to find POIs by name and to select POIs in cases when they appear too small (device far from the map) or when many POIs are clustered together. Button (5) shows a popup panel, which allows the user to filter the POIs by category. Buttons (6) and (7) allow control over the temporal con- 
text colour-coding, by allowing users to display popularity information for specified days of the week and times (hours) of the day, selectable through drop down lists. Button (8) refreshes the information each time a user selects different values from the drop down buttons. Finally, button (9) is used to find the location of the user if the application did not succeed in finding it automatically. The navigation panel in the bottom of the screen (10) provides navigation details to the user, such as the name of the destination, the estimated time to arrival and the remaining walking distance.
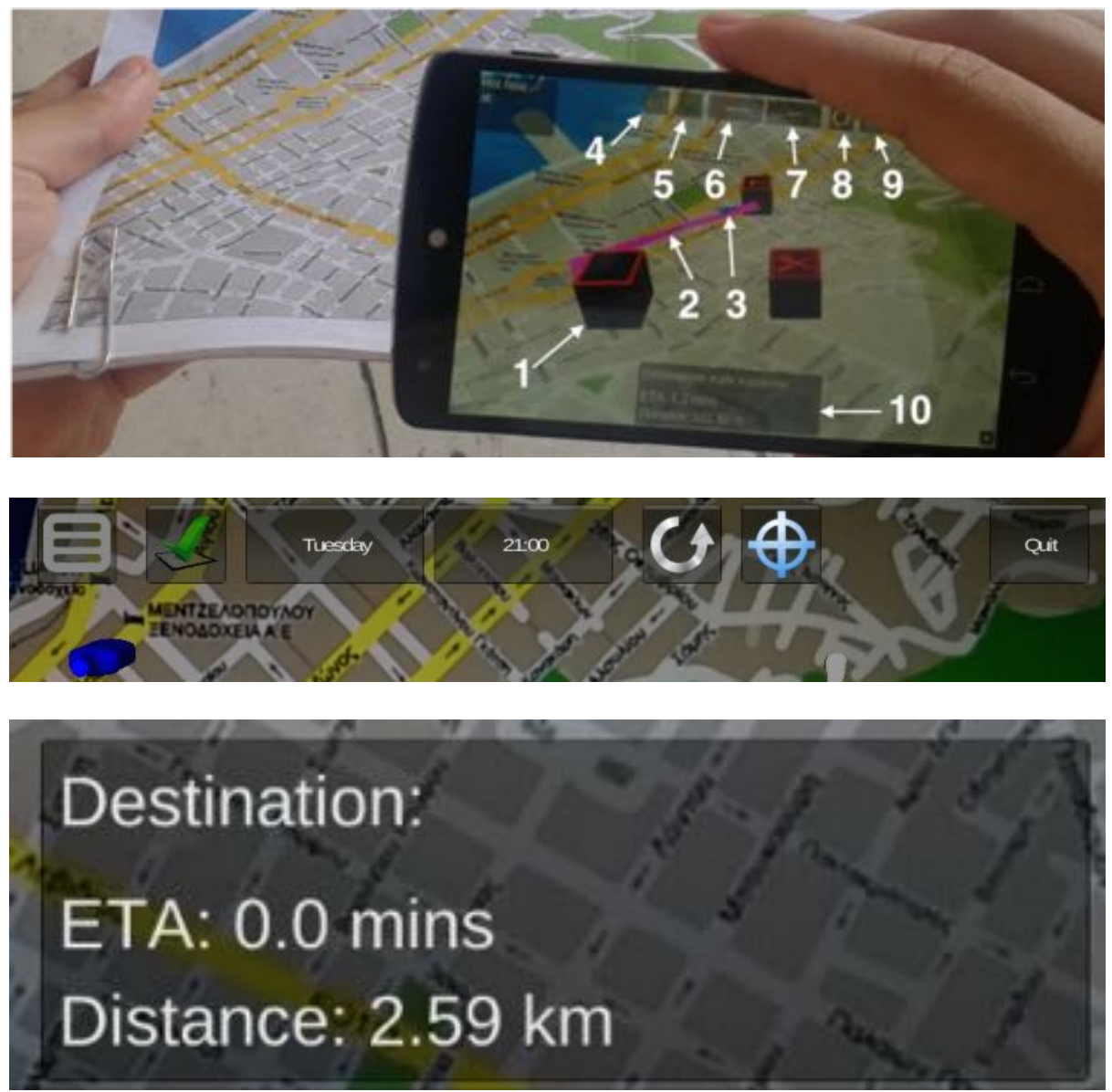

Fig. 1. The HoloPlane AR Interface working with a paper map (top), detailed view of the top bar UI controls (middle) and navigation panel (bottom) 


\section{$4 \quad$ Evaluation}

\section{Experiment Design}

Our participants were 23 undergraduate engineering students from various disciplines (14 male, 9 female), from 17 European countries, who were visiting the city of Patras for a summer school. Their ages ranged between 18 and 26 years old and none had previous experience with mobile AR applications. All participants mentioned familiarity with navigation applications, with $40 \%$ stating frequent use and $17 \%$ indicated always using just a mobile application while visiting a new place. We found a low preference for fixed city maps (e.g. wall-mounted) and paper maps $(22 \%$ in both cases) compared to mobile navigation apps. To establish thus a baseline that would be representative of our participants' usual behaviour, we chose to compare our prototype to the most preferable navigation aid for our participants, i.e. a mobile navigation app and not a paper map. Hence we selected the familiar navigation tool installed on all Android devices, i.e. Google Maps (GM). For the field experiment we provided participants with four devices of equivalent capabilities in terms of processor speed and screen size (LG Nexus 4 and Nexus 5 and Samsung S3 and S4), which all ran our application with good performance. In order to test our prototype in navigation tasks, we established two routes of equal complexity in terms of turns and walking distance (Figure 2), requiring approximately 10 minutes of walking time from a person familiar with the area. We let participants split themselves into 8 groups, allowing friends to work together to better simulate real tourist groups - the first four groups completed the first route using the HoloPlane AR prototype and proceeded to GM navigation for the second route. This order was reversed for the remaining four groups. Each team was accompanied by a researcher who knew the routes and was able to provide help if the team did not succeed to find the destination. Finally, in each team, one user volunteered to control the device and map (where used), while the other two participants were termed as "companions" and were instructed to ask for control of the device and map, if they so desired. This setup is representative of situations where one person assumes the navigator's role, typically because they own the device. As stated previously, HoloPlane is designed to be used with any simple printed map. For our experiment, we provided participants with a colour printed map from the Google Maps website that shows the experiment area at a scale of roughly 1: 18055 (zoom level 16). This is the smallest scale at which Google shows names for all streets and not just major ones. Furthermore, this scale allows the map to depict as wide an area as possible, maintaining label readability for the users. We selected an A4 print size, to represent a typical situation for users who might have printed a map at home before travelling, or during their stay (e.g. at Internet café), as few users would typically have access to a large format printer such as A3 or larger.

\section{Data Collection}

We collected GPS positioning data for each team. The researchers, who accompanied each team, also noted the number of times participants stopped to consult the 
application and make a route choice during navigation. At the end of each navigation task we asked each participant to complete a NASA TLX questionnaire, so that we could obtain their subjective workload impression. We also asked them to complete two validated questionnaires for each system: a Brief Mood Introspection Scale (BMIS) [9] in order to measure mood and a User Experience Questionnaire (UEQ) [8] for their overall experience.

\section{Results}

This section reports our observations based on the quantitative and qualitative results. The tests reported in this section were chosen according to the outcomes of normality tests on all our variables.

\section{Quantitative measures.}

In Figure 2, we show the participants' walking behaviour during navigation, which is visualized through a heatmap-based depiction of GPS traces. We report this data as recorded by the device GPS without statistical significance analysis, since the number of teams was too small to provide an adequate sample size for statistical significance.

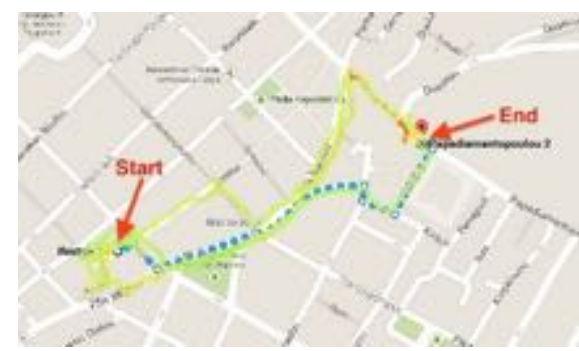

Route $1(\mathrm{GM})$

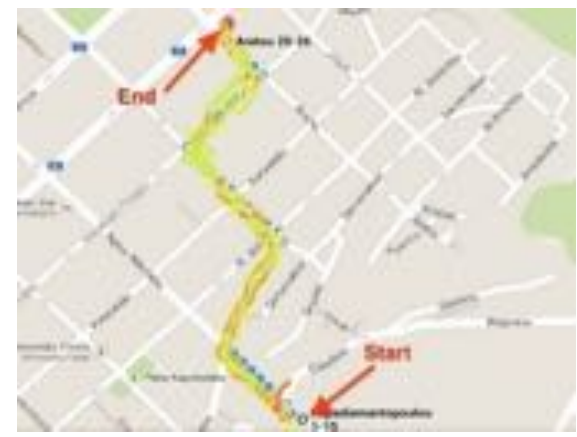

Route 2 (GM)

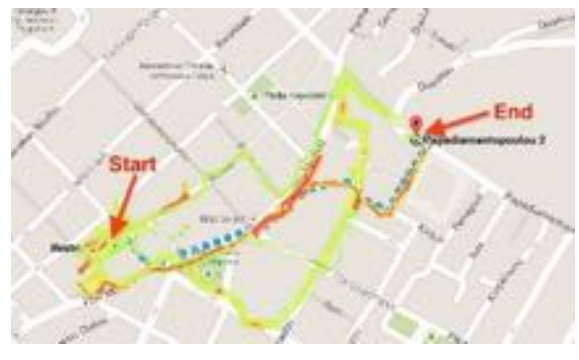

Route 1 (HP)



Route 2 (HP)

Fig. 2. Participant Routes and heatmapped GPS traces. The red segments show where participant speed was less than $1 \mathrm{~km} / \mathrm{h}$ 
Overall teams took less time to navigate with $\mathrm{GM}(\mathrm{mgm}=896.96 \mathrm{~s}$, sd $\mathrm{gm}=295.93 \mathrm{~s}$, $\left.\mathrm{mhp}_{\mathrm{h}}=1093.93 \mathrm{~s}, \mathrm{sdhp}=319.10 \mathrm{~s}\right)$. However with GM they made more stops to consult the tool $(\mathrm{mgm}=8.75, \mathrm{sd} \mathrm{gm}=9.77, \mathrm{mhp} 7.88, \mathrm{sdhp}=3.41)$. We measured the length of pauses they made during navigation, i.e. periods longer than 5 seconds where the speed was less than $1 \mathrm{~km} / \mathrm{h}$. There were fewer such periods with $\mathrm{GM}(\mathrm{mgm}=10.86, \operatorname{sdgm}=3.31$, $\left.\mathrm{mhp}_{\mathrm{n}}=17.86, \mathrm{sdhp}=7.22\right)$ which lasted also less time $\left(\mathrm{m}_{\mathrm{gm}}=273.88, \mathrm{dgm}_{\mathrm{g}}=241.19\right.$, $\mathrm{mhp}_{\mathrm{h}}=316.87, \mathrm{sdhp}=178.0$ ). Finally, in terms of distance covered, this was less with GM $\left(\mathrm{m}_{\mathrm{gm}}=690.80 \mathrm{~m}, \mathrm{sdgm}=81.01 \mathrm{~m}, \mathrm{mhp}=842.79 \mathrm{~m}, \mathrm{sdhp}=192.8 \mathrm{~m}\right)$.

\section{Participant Workload Assessment}

At the end of each navigation task, we issued each participant with a NASA-TLX questionnaire to obtain their subjective ratings of their experience with each navigation tool. The overall results are summarized in Figure 3. Overall it can be seen that GM was rated better than our prototype (a lower score is better), with the exception of physical effort. The latter is expected, as the routes were carefully chosen to present equal levels of walking difficulty and length. Concerning the remaining five variables, a statistical significance in the difference of means was only found for effort to complete the task, using a paired-sample T-test $\left(\mathrm{m}_{\mathrm{gm}}=7.61, \mathrm{sd}_{\mathrm{gm}}=4.878, \mathrm{~m}_{\mathrm{hp}}=10\right.$, $\left.\mathrm{sd}_{\mathrm{hp}}=3.357, \mathrm{p}<0.05\right)$ and performance, using a Wilcoxon signed rank test $\left(\mathrm{m}_{\mathrm{gm}}=3.43\right.$, $\left.\mathrm{sd}_{\mathrm{gm}}=3.287, \mathrm{~m}_{\mathrm{hp}}=6.26, \mathrm{sd}_{\mathrm{hp}}=4.826, \mathrm{p}<0.05\right)$. Overall thus it appears that the GM tool led to the expenditure of less effort to complete the navigation task and participants felt more successful using it.

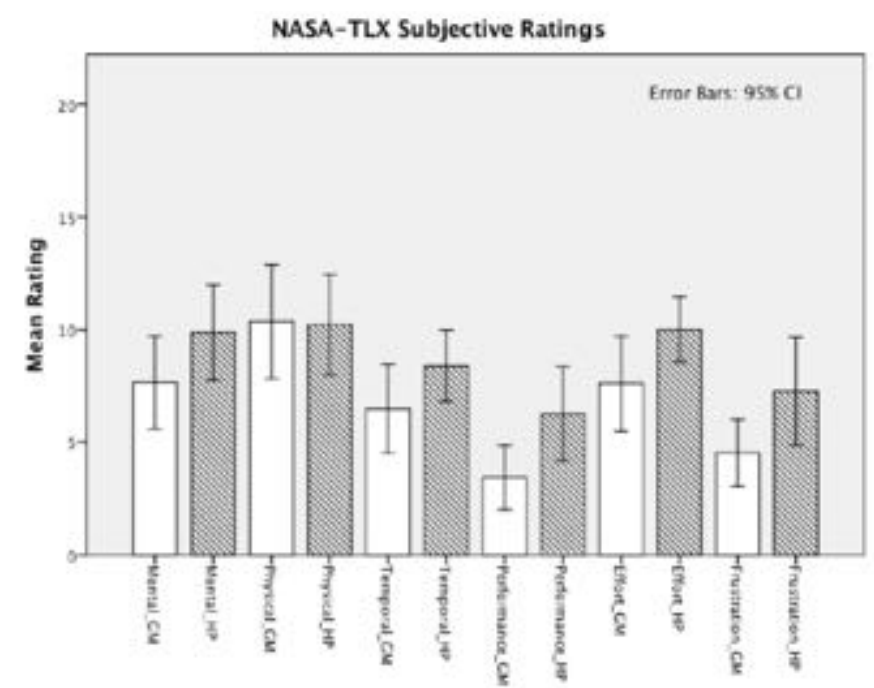

Fig. 3. Subjective Workload Assessment

\section{Participant Affective State}

Using the BMIS questionnaire at the end of each task, we asked participants to give us insight to their affective state during the tasks. This questionnaire contains 16 ad- 
jectives describing affective state. Before letting the participants answer the questionnaire, we explained in detail each adjective, in order to be sure that they fully understood the choices and their meaning. The analysis of the user responses was made on the Calm - Arousal and Unpleasant - Pleasant axes, and is depicted below in Figure 4. It can be generally seen that the participants' experience was rated positively in terms of pleasantness and that participants felt averagely aroused during the navigation tasks.



Fig. 4. Affective state during navigation tool use

Further analysis reveals that when considering all users, no statistically significant differences using Wilcoxon signed rank tests for the two navigation tools, on either the Unpleasant-Pleasant $\left(\mathrm{m}_{\mathrm{gm}}=9.22, \mathrm{sd}_{\mathrm{gm}}=4.69, \mathrm{~m}_{\mathrm{hp}}=7.44, \mathrm{sd}_{\mathrm{hp}}=5.67\right)$ or the CalmArousal axis $\left(\mathrm{m}_{\mathrm{gm}}=14.96, \mathrm{sd}_{\mathrm{gm}}=3.28, \mathrm{~m}_{\mathrm{hp}}=15.87, \mathrm{sd}_{\mathrm{hp}}=4.38\right)$. We went further by breaking up the users according to their roles (app users and companions) and analyzing the respective data. We did not find any statistically significant differences using Wilcoxon signed rank tests in either axis for any of these user categories. Given the previously found statistically significant difference in performance, we conclude that while the participants believe they fared worse with the HoloPlane prototype, nevertheless, their experience was just as pleasant as with GM.

\section{Participant User Experience.}

At the end of each navigation task, we asked each participant to complete the User Experience Questionnaire, in order to obtain a measure of their assessment of each navigation tool. The questionnaire generally assumes a positive appraisal on each dimension if the mean exceeds 0.8 , or a negative appraisal if the mean is less than 0.8 . Analysis with Wilcoxon signed rank tests reveals that statistically significant differences appear only in the dimensions of perceived Efficiency $\left(\mathrm{m}_{\mathrm{gm}}=1.978, \mathrm{sd}_{\mathrm{gm}}=0.170\right.$, $\left.\mathrm{m}_{\mathrm{hp}}=0.578, \mathrm{sd}_{\mathrm{hp}}=0.875, \mathrm{p}<0.01\right)$, Dependability $\left(\mathrm{m}_{\mathrm{gm}}=1.674, \mathrm{sd}_{\mathrm{gm}}=0.82, \mathrm{~m}_{\mathrm{hp}}=0.924\right.$, $\left.\mathrm{sd}_{\mathrm{hp}}=0.89, \mathrm{p}<0.05\right)$, Stimulation $\left(\mathrm{m}_{\mathrm{gm}}=0.728, \mathrm{sd}_{\mathrm{gm}}=1.047, \mathrm{~m}_{\mathrm{hp}}=1.467, \mathrm{sd}_{\mathrm{hp}}=0.728\right.$, $\mathrm{p}<0.01)$ and Novelty $\left(\mathrm{m}_{\mathrm{gm}}=-0.63, \mathrm{sd}_{\mathrm{gm}}=1.297, \mathrm{~m}_{\mathrm{hp}}=1.609, \mathrm{sd}_{\mathrm{hp}}=0.856, \mathrm{p}<0.01\right)$. These 
outcomes for the Stimulation, Efficiency and Dependability are in line with the outcomes from our previous questionnaires. The observed difference in Stimulation measures is somewhat unexpectedly in disagreement with the parity observed in the Arousal-Calm axis earlier. However, a more careful inspection of the wording of the UEQ adjectives used to measure on the positive scale for this axis, uncovers that these imply a level of engagement, instead of measuring affective state (valuable, exciting, interesting, motivating). Finally, there is clear indication here that our participants considered HoloPlane to present significant novelty.

\section{Other observations}

When observing participant bodily configuration, we noticed a more relaxed approach with the AR tool, compared to "squeezing in" to view the device instructions when using GM, an observation also made in [11]. In Figure 5, we show several examples of use of the HoloPlane prototype. In these, the shared use of the hybrid working space is evident in several collaboration examples: In the first (Figure 5a), the "navigator" has control of both the paper map and the device. Companions are gathered around the map, paying attention to the printed surface which is clearly visible and intelligible to all, while the screen of the device is used only by the navigator. His role here is to communicate what he sees on the device, to the companions, so that a shared understanding can be achieved. Communication is verbal, since both the navigator's hands are occupied. In the second example (Figure 5b), the "navigator" controls the device, while one of the companions is holding the map. Here, the "navigator" is seen to be pointing on the map, in order to communicate to the companions his knowledge in a more comprehensible manner. This mode of communication is more direct and helps companions understand more easily what the navigator sees. Finally, in Figure 5c, we note that the communication of spatial awareness is initiated by the companion, who is holding the paper map and at the same time pointing to a location on it. At the same time, the navigator is trying to understand the companion's communication and match it to what is represented on the device screen. This example shows that the hybrid system allows for more active participation in the navigation task by all group members.



(a)

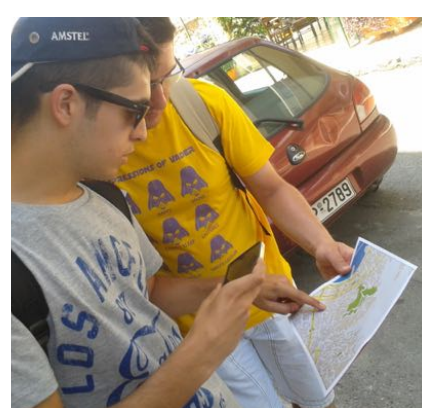

(b)

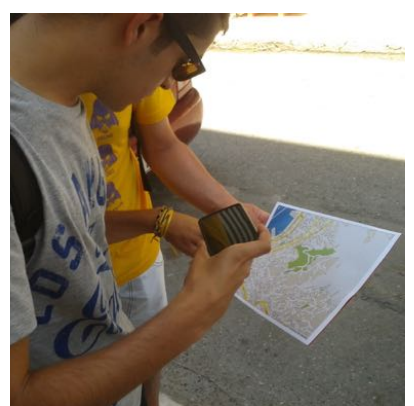

(c)

Fig. 5. Group behaviour during use of the Holoplane hybrid interface 
The next figure (Figure 6) shows some instances of the navigation task, during use of the Google Maps interface. Here it is easy to observe that the planning task is made much more difficult for all users, since the screen real-estate is quite small and participants have to gather tightly to see what is displayed. Not all participants are able to point to the screen in order to communicate their understanding, hence limiting their ability to make a contribution to the planning (Figure 6a). During transit to established waypoints, the companions often resigned to being simple followers (Figure $6 \mathrm{~b})$. Here, the companion on the right is talking to the navigator, since they were able to plan the route together previously, leaving the female companion unable to contribute to the planning. The female companion, adopts a passive mode since she did not participate in the planning stage, and is seen to be walking just ahead of the group, keeping an ear out for the navigator's next instruction. This is evident also in Figure $6 \mathrm{c}$, where the female companion is simply looking around. The navigator is ahead of the group on his own, trying to determine the group's whereabouts, while the male companion is trying to visually match the surrounding location to the printout of the navigation target given to the group.

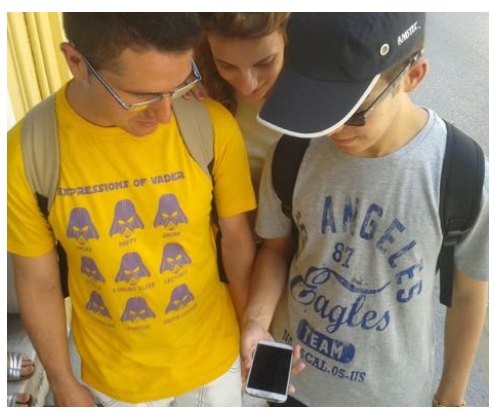

(a)



(b)

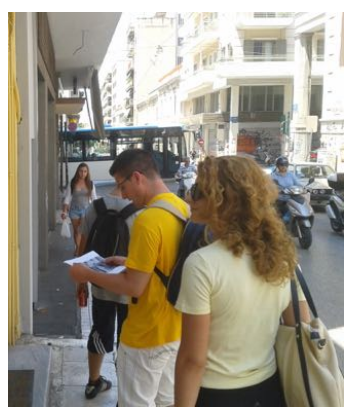

(c)

Fig. 6. Group behaviour during use of the Google Maps interface

\section{Discussion and Future Work}

Our evaluation was based on the use of validated questionnaires whose use is not widespread in the field of mobile HCI. This approach contrasts previous research in [10] and [11] whose findings are based on the analysis of qualitative interviews. Yet, our preliminary evaluation did not find any significant performance advantages of augmenting a paper map for navigation, a result that is completely in line with [10] and [11]. This outcome provides indication that the questionnaire-based approach has merit and can be used effectively in the place of qualitative interviews, where the danger of researcher bias in the analysis of results is significant. Another similarity with [11] is that when observing participant bodily configuration, we noticed a more relaxed approach with the AR tool, compared to "squeezing in" to view the device instructions when using GM (Figure 5). 




Fig. 7. User experience during navigation tool use

The reason why no advantages were observed with the AR interface may relate to the size of the augmented paper map. We selected a relatively small printed area (A4) to represent a typical situation of users printing their own maps. Perhaps a larger shared map might make the magic lens interface more usable, as suggested by [6], although there, maps were fixed on to a wall surface, where as in our scenario users have to be able to conveniently hold the map. Hence, while providing a larger printed map might make its augmentation more usable, it might detract from its key benefit (i.e. portability and manipulability). A further consideration for performance is item density: In our situation, the item density was very low and included just two POIs and the route. As per [16], it can be expected that our users might have focused more on the paper map than the magic lens, hence preventing the system from achieving its performance potential. Further tests with different item densities (e.g. routes with multiple waypoints) would be needed to verify any effects.

As indicated by the Stimulation axis in the UEQ, our participants felt more engaged as group members with the HP system than GM, where a single user takes on the role of the navigator and collaboration is hindered, as the small screen limits the information space. The reported level of engagement might be an effect of the high perceived novelty of the system, since both axes (Stimulation \& Novelty) relate to hedonic quality perception. However, the UEQ Novelty axis has been found not to correlate with the Stimulation axis in other research [18]. As a side effect of increased engagement with the navigation task, the acquisition of spatial knowledge for all users might be improved for users as per [21], but further tests would be needed.

It is encouraging that participants found the AR tool just as attractive as the standard navigation tools. The issues of mental workload and efficiency appraisals can be attributed to the novelty and unfamiliarity of our application to users.

To this end, we are hoping to conduct further, more extensive trials to eliminate familiarity factors from the results. Furthermore, given that augmented maps can be 
used as a collaboration tool, our future research will also encompass the use of our AR tool with public displays of maps.

\section{References}

1. Adams, A. and Cox, A. L. 2008. Questionnaires, indepth interviews and focus groups. In Research Methods for Human Computer Interaction, Cairns, P. and Cox, A. L. (eds.). Cambridge University Press, Cambridge, UK, 17-34

2. Bouwer, A., Nack, F. and Evers, V. 2011. Towards support for collaborative navigation in complex indoor environments. In Proc. CSCW'11, 601-604.

3. Brown, B., \& Chalmers, M. 2003. Tourism and mobile technology. 2003. In Proc. ECSCW'03, 335-354

4. Čopič Pucihar, K., Coulton, P. and Alexander, J. 2014. The use of surrounding visual context in handheld AR: device vs. user perspective rendering. In Proc. CHI'14, 197-206.

5. Dünser, A., Billinghurst, M., Wen, J., Lehtinen, V. and Nurminen, A. 2012. Exploring the use of handheld AR for outdoor navigation. Computers \& Graphics, 36, 8: 1084-1095.

6. Grubert, J., Pahud, M., Grasset, R., Schmalstieg, D. and Seichter, H. 2014. The utility of Magic Lens interfaces on handheld devices for touristic map navigation. Pervasive and Mobile Computing, 18, 88103.

7. Komninos, A., Stefanis, V., Plessas, A. and Besharat, J. 2013. Cloud-based capture and sharing of urban dynamics using scarce check-in data. IEEE Pervasive Computing, 12, 4: 20-28.

8. Laugwitz, B., Held, T., \& Schrepp, M. 2008. Construction and evaluation of a user experience questionnaire. In HCI and Usability for Education and Work, Holzinger, A. (ed.), Springer, Heidelberg, Germany, 63-76.

9. Mayer, J. D. and Gaschke, Y. N. 1988. The experience and meta-experience of mood. Journal of Personality and Social Psychology, 55: 102-111.

10. Morrison, A., Mulloni, A., Lemmelä, S., Oulasvirta, A., Jacucci, G., Peltonen, P. and Regenbrecht, H. 2011. Collaborative use of mobile augmented reality with paper maps. Computers \& Graphics, 35, 4: 789-799.

11. Morrison, A., Oulasvirta, A., Peltonen, P., Lemmela, S., Jacucci, G., Reitmayr, G. and Juustila, A. 2009. Like bees around the hive: a comparative study of a mobile augmented reality map. In Proc. CHI'09, 1889-1898

12. Müller, J., Jentsch, M., Kray, C. and Krüger, A. 2008. Exploring factors that influence the combined use of mobile devices and public displays for pedestrian navigation. In Proc. NordiCHI'08, 308-317.

13. Norrie, M. and Signer, B. Overlaying paper maps with digital information services for tourists. 2005. In Proc. ICTT'05, 23-33.

14. Raita, E. User interviews revisited: identifying user positions and system interpretations. 2012. In Proc. NordiCHI'12, 675-682

15. Reilly, D., Mackay, B., Watters, C. and Inkpen, K. 2009. Planners, navigators, and pragmatists: collaborative wayfinding using a single mobile phone. Personal and Ubiquitous Computing, 13, 4: 321-329.

16. Rohs, M., Schöning, J., Raubal, M., Essl, G. and Krüger, A. 2007. Map navigation with mobile devices: virtual versus physical movement with and without visual context. In Proc. ICMI '07, 146-153.

17. Stroila, M., Mays, J., Gale, B. and Bach, J. 2011. Augmented transit maps. In Proc. WACV'11, 485-490 